\title{
FATIGUE FAILURE BY FLOW-INDUCED VIBRATION
}

\author{
Y.Murakami' and M.Inoue' and A.Sueoka' \\ S.Odahara' and M.Kobayashi2and N.Fujiwara3 \\ ${ }^{\text {I }}$ Department of Mechanical Science and Engineering, Kyushu University, 6-10-1, \\ Hakozaki, Higashi-ku, Fukuoka \\ * Fukuoka Nursing School Attached to National Kyushu Medical Center Hospital, \\ 2-5-18, Najima, Higashi-ku, Fukuoka \\ ${ }^{3}$ SumitomoConstruction Machinery, 1-1, Maeda, Niihama-shi, Ehime
}

\begin{abstract}
The mechanism of fatigue failure caused by flow-induced vibration was studied by using a small wind tunnel. The necessary conditions of flow-induced vibration for a $0.45 \%$ carbon steel specimen attached to a larger Styrofoam cylinder were investigated. Possible methods of detecting the symptom of fatigue crack initiation and propagation were sought. The change in natural frequency and displacement amplitude of the test cylinder during fatigue test was investigated. The strain history at the anticipated crack initiation sites was monitored. Specimens containing an artificial small hole were used to localize the crack initiation site. A small portable service strain histogram recorder developed in another project was used to monitor the variation of strains due to crack growth from the small hole. Fatigue damage accumulation based on Miner's rule was calculated and compared with the experimental results.
\end{abstract}

\section{INTRODUCTION}

The liquid sodium leakage accident which happened in the fast breeder reactor, MONJU on 8th December 1995 is one of the most serious accidents related to nuclear reactor in Japan. The cause of this accident was the fatigue failure caused by flow-induced vibration of a thermometer fixed in the pipe of the secondary cooling system with liquid sodium [1]. Recently, 6th July 1999, the traffic sign pole of emergency parking area and an emergency telephone guidance pole (weight $300 \mathrm{~kg}$ ) at the Tokyo urban highway were broken, and dropped onto the city road under the highway and damaged a car running. The cause of accident in the case was also fatigue failure by flow- 
induced vibration of a traffic sign pole in strong wind [2]. Several examples of fatigue failure by flow-induced vibration have been reported in the past [3-51. According to the authors' survey, there is no research paper reporting the mechanism of fatigue failure by laboratory flow-induced tests. The aim of this study is to clarify the mechanism of fatigue failure by flow-induced vibration. First, the conditions for exciting flow-induced vibration were analyzed. A small wind tunnel for fatigue failure by flow-induced vibration was produced. In order to detect fatigue crack in the early stage of test, we paid particular attention to three parameters: (1) variation of natural frequency, (2) variation of displacement amplitude of the specimen system, (3) variation of strain histogram at the anticipated crack initiation site. An artificial small hole was introduced to the specimens so that monitoring crack growth behavior became easy. A small portable service strain histogram recorder (Mini Rainflow Corder, MRC $[6,7])$ developed in another project was used to monitor the relationship between crack growth and the variation of strain frequency near the artificial small hole. Natural frequency was determined from the data of MRC. The displacement amplitude of test cylinder was measured from the picture taken by video camera. The strain histogram was acquired directly by the MRC. Fatigue life was predicted based on Miner's rule [S] by using the data at the early stage of test.

\section{MATERIAL AND TEST PROCEDURE}

The material used was $0.45 \mathrm{C}$ steel turned after annealing at $844^{\circ} \mathrm{C}$ for $1 \mathrm{hr}$. The chemical composition was (wt. \%) $0.47 \mathrm{C}, 0.21 \mathrm{Si}, 0.82 \mathrm{Mn}, \mathrm{O} . \mathrm{OlSP}, \mathrm{O} . \mathrm{O} 18 \mathrm{~S}, \mathrm{O} . \mathrm{OlCu}, 0.018 \mathrm{Ni}$ and $0.064 \mathrm{Cr}$. The mechanical properties were $620 \mathrm{MPa}$ tensile strength, 339MPa lower yield strength, 1105MPatrue fracture strength and $\mathbf{5 3 . 8 \%}$ reduction of area. Figure 1(a) shows the shape and dimensions of the specimen which contains a drilled small hole of $200 \mu \mathrm{m}$ diameter and depth at the minimum cross section. Specimens were then annealed in a vacuum at $600^{\circ} \mathrm{C}$ for $1 \mathrm{hr}$ to relieve residual stress caused by drilling. The Vickers hardness after vacuum annealing was HP178 which is a mean value of each specimen measured at 4 points with load of $0.98 \mathrm{~N}$. The scatter in $\boldsymbol{H V}$ was within $5 \%$. Figure 1(b) shows the configuration of the wind tunnel made in this study. The suction fan was equipped with a lattice net attached at the entry of a fan in order to reduce swirl velocity and to obtain a uniform flow around the test cylinder. The test cylinder consists of a core cylinder of S45C steel partially covered with a Styrofoam cylinder and polyurethane, a specimen and a base. The wind velocity in the wind tunnel can be controlled in the range from $0 \mathrm{~m} / \mathrm{s}$ to $12 \mathrm{~m} / \mathrm{s}$. The measurements were made at every $30 \mathrm{~min}$ from start to specimen failure. Strain histogram history was measured by MRC based on the rainflow algorithm for last $5 \mathrm{~min}$ in an every 30 min measurement block. Vibration behavior of the test cylinder was recorded for lmin by video camera from the top of the wind tunnel. After stopping the fan at every $30 \mathrm{~min}$, crack length was measured by the replica method. This procedure was repeated until final failure of specimen.

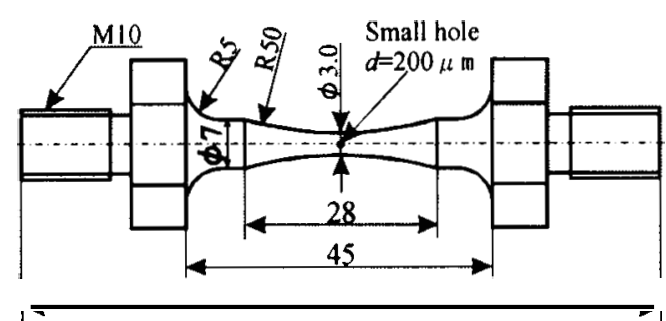

(a) Shape and dimensions of specimen.

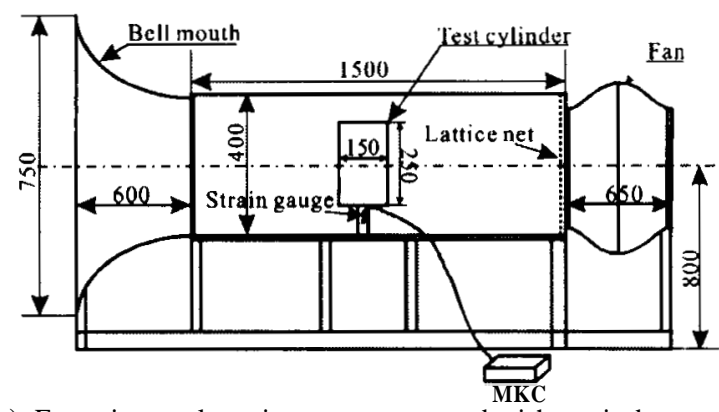

(b) Experimental equipment composed with a wind tunnel, specimen and a small portable strain recorder. $(\mathrm{mm})$

Figure 1: Shape and dimensions of specimen and Experimental equipment 
The Strouhal number, $\mathrm{S}$, controls the condition of flow-induced vibration. Figure 2 shows $S$ must be in the specific ranges to obtain flow-induced vibration [9]. $S$ is defined by

$$
S=f D / U
$$

, where $f_{s}$ is von Karman vortex shedding frequency in $\mathbf{H z}, \mathrm{D}$ the diameter of test cylinder in $\mathrm{m}, U$ free flow velocity in $\mathrm{m} / \mathrm{s}$. In the case of the MONJU accident, $f_{n}=265 \mathrm{~Hz}, D=0.01 \mathrm{~m}, U=5.0 \mathrm{~m} / \mathrm{s}$ and $S=0.53$ which corresponds to the domain No.3 of Fig.2 [10]. Therefore, symmetric vortices shed from both sides of the thermometer sheath, and the thermometer sheath vibrated parallel to flow direction [10]. The lift force acting on test cylinder per unit length is expressed by

$$
F=\boldsymbol{p} U^{2} D C_{L} / 2
$$

where $p$ is fluid density in $\mathrm{kg} / \mathrm{m}^{3}$, and $\mathrm{C}$, lift coefficient. From the viewpoint of fatigue crack observation, air was used as fluid for wind tunnel. The density of air is approximately 111000 of water. Although the condition of $U=5.0 \mathrm{~m} / \mathrm{s}$ and $S=0.37$ corresponds to the domain of No.2 of Fig.2, fatigue crack is not expected to initiate due to small lift force calculated by Eqn.2. Thus, the condition of the domain No.1 in which large amplitude can be obtained as adopted. On the other hand, von Karman vortex shedding frequency must agree with natural frequency of test cylinder. In this study S 0.17 was adopted to obtain the largest amplitude of cylinder in the domain No.1. The condition leading fatigue failure for short time was found by preliminary tests in which several shapes and dimensions of test cylinders and specimens, and free flow velocity were examined. Thus, the test condition was finally determined as $f_{s}=f_{n}=10.8 \mathrm{~Hz}, D=0.15 \mathrm{~m}$ and $U=9.7 \mathrm{~m} / \mathrm{s}$.
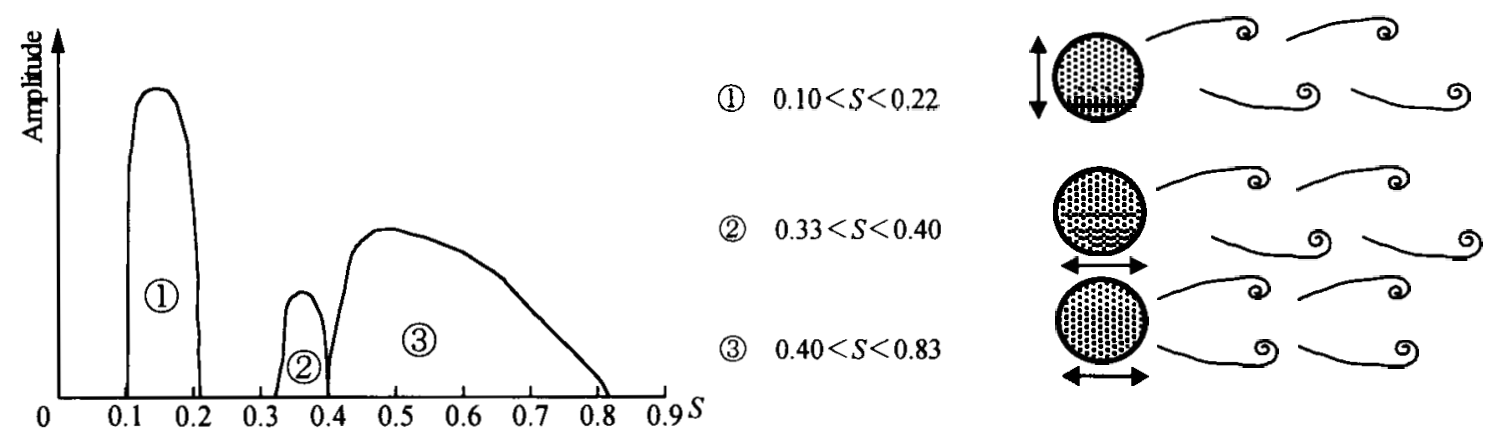

Figure 2 :The relationship betweenS (Strouhal number) and amplitude(Blevins ${ }^{(9)}$ ). (1) is the domain of the vibration transverse to flow direction. (2) and (3) are the domain of the vibration parallel to flow direction. Vortex shape changes depending on Strouhal number.

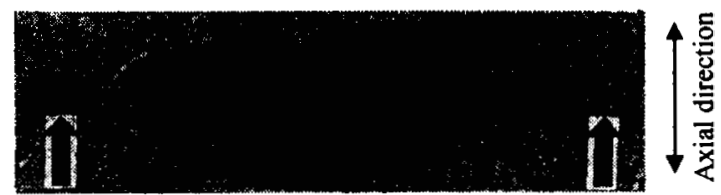

Figure 3 :Crack at small hole after $N=1.3 \times 10^{6}$ cycles. Crack length $l=723 \mu \mathbf{m}$.

The portable strain histogram recorder, MRC, was used for data acquisition of strain history. Figure 3 shows crack propagation almost perpendicular to the axis of specimen. Strain gauge No.1 was attached to the site where the variation of strain history due to crack initiation and propagation were expected. Figure 4 shows the positions of strain gauges No.1 and No.3 in relation with the small hole. The distance from the center of the small hole to Strain gauge No. 1 was $1660 \mu \mathrm{m}$. Strain gauge No.3 was attached at the opposite side of specimen with respect to the small hole. MRC counts strain frequency by the Rainflow counting algorithm. The maximum strain range 


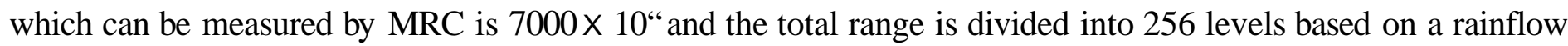
algorithm. The acquired data were analyzed by a personal computer.

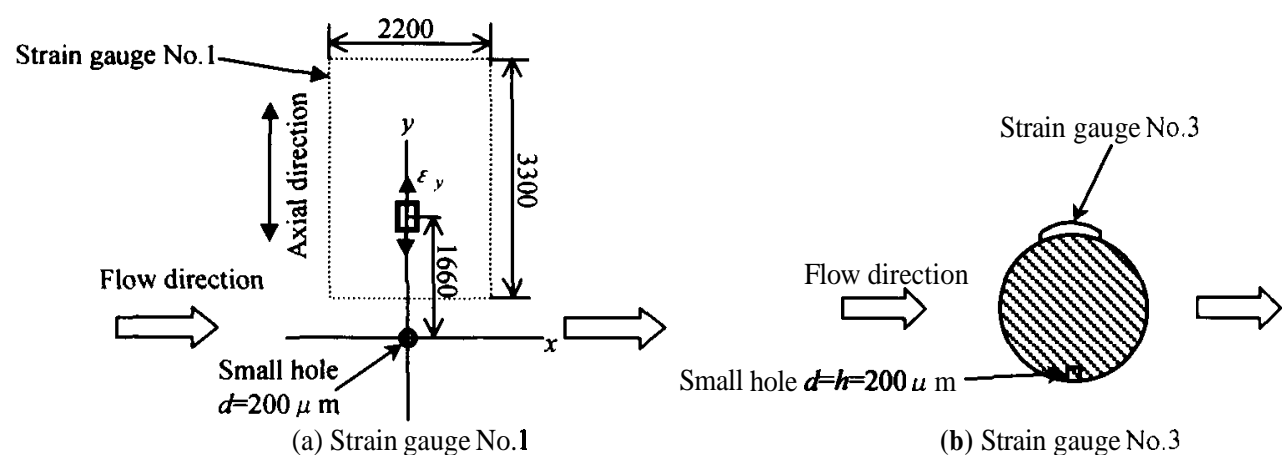

Figure 4 :Strain gauge positions with respect to the location of the small hole $(\mu \mathrm{m})$. (a) Strain gauge No.1 viewed from horizontal direction. (b) Strain gauge No.3 at the opposite side to the small hole.

\section{RESULT AND DISCUSSION}

\section{Trace of the vibration of test cylinder}

Vibration behaviour of test cylinder recorded by video camera from the top of the wind tunnel. A mark was put at the top of the test cylinder. Figure 5(a) illustrates the observation method. Figure 5(b) and (c) shows the observed trace of the mark at the beginning of the test and after $1260 \mathrm{~min}$. The vibration behaviour is extremely irregular. Figure 5(b) shows the vibration behaviour after the first $\mathbf{3 0}$ min interval when no crack exists. The direction of vibration is almost perpendicular to flow direction. The variation behaviour of test cylinder approximately belongs to the condition of Fig.2. Figure 5(c) shows the vibration behaviour after 1260 min testing when the crack has length $l=1110 \mu \mathrm{m}$. There is no apparent difference between the amplitude and vibration direction of Fig.5(b) and (c). It follows that the amplitude of cylinder hardly changes until crack grows to $1 \mathrm{~mm}$ and it is very difficult to predict the symptom of fatigue failure by vibration amplitude.

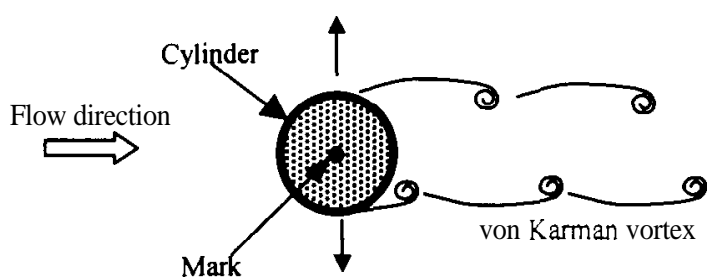

(a) Observation mark at the top of test cylinder

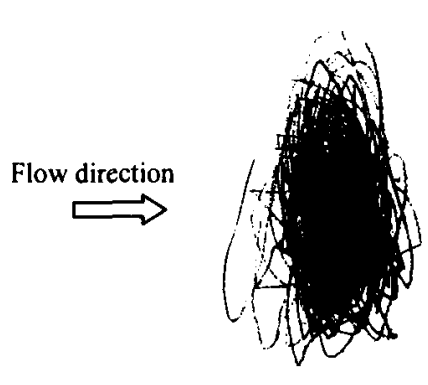

(b) The first $\mathbf{3 0} \mathrm{min}$

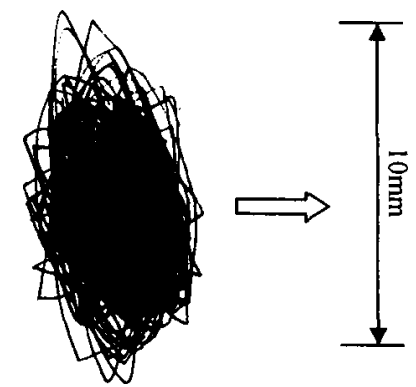

(c) After $1260 \mathrm{~min}$, crack length $l=1110 \mu \mathrm{m}$ Figure 5 :Trace of the mark during the last $1 \mathrm{~min}$ at the measurement interval, $\mathbf{3 0} \mathrm{min}$. (b) After first 30 min interval with no crack, (c) After 1260 min test intervals with crack. Crack length $l=1110 \mu \mathrm{m}$.

\section{Variation of naturalfrequency}

Variation of natural frequency with crack growth may be thought to capture the symptom of fatigue failure by flow-induced vibration. Natural frequency is defined from strain frequency for 5 min measurement by MRC at strain gauge No.3. Number of cycles $N$ is defined by those of reversals based on the Rainflow algorithm [11,121 and as twice as ordinary counts. Thus the natural frequency $f_{n}(\mathrm{~Hz})$ is defined by

$$
\mathbf{S}^{\prime \prime}=\frac{\text { Strain frequency measured at strain gauge No.3 for } 5 \mathrm{~min} / 2}{5 \times 60 \mathrm{sec}}
$$


Figure 6 shows the relationship between variation of natural frequencyf, $(\mathbf{H z})$ and crack length $l(\mu \mathrm{m})$. The crack initiated at the edge of the hole at $N_{c}=3.5 \times 10^{5}$ cycles and the specimen failed at $N_{f}=2.3 \times 10^{6}$ cycles. However, natural frequency hardly varied almost until final fracture. Particularly, the lack of change of natural frequency at crack length $1 \mathrm{~mm}$, demonstrates the difficulty of predicting the beginning of fatigue failure at an early stage of the test $[1,13,14]$.

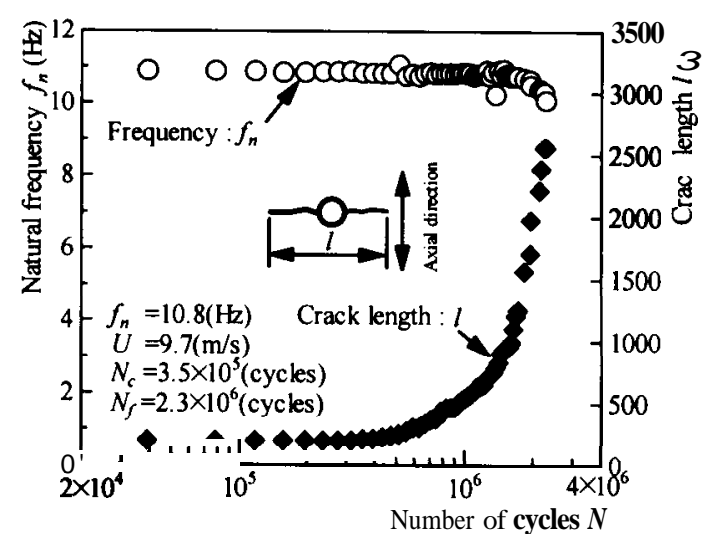

Figure 6 :Variation of natural frequency and crack growth. $f_{n}$ : Natural frequency. $l$ : Crack length. $N_{c}$ : Cycles to crack initiation, $N_{f}$ : Cycles to failure.

\section{Strain frequen $c y$ distribution}

Variation of strain frequency distribution investigated to capture a symptom of fatigue failure when the crack size was small (less than $1 \mathrm{~mm}$ ). Figure 7(a) and (b) shows the strain frequency distributionsmeasured by MRC. In these histogram, the abscissa is strain $E$, the ordinate is strain frequency $n$ for 5 min measurement. Figure 7(a) shows strain frequency distribution measured at strain gauge No.3. This strain gauge was attached at the opposite side of specimen with respect to the small hole (Fig.4(b)). In this histogram the strain frequency distribution hardly
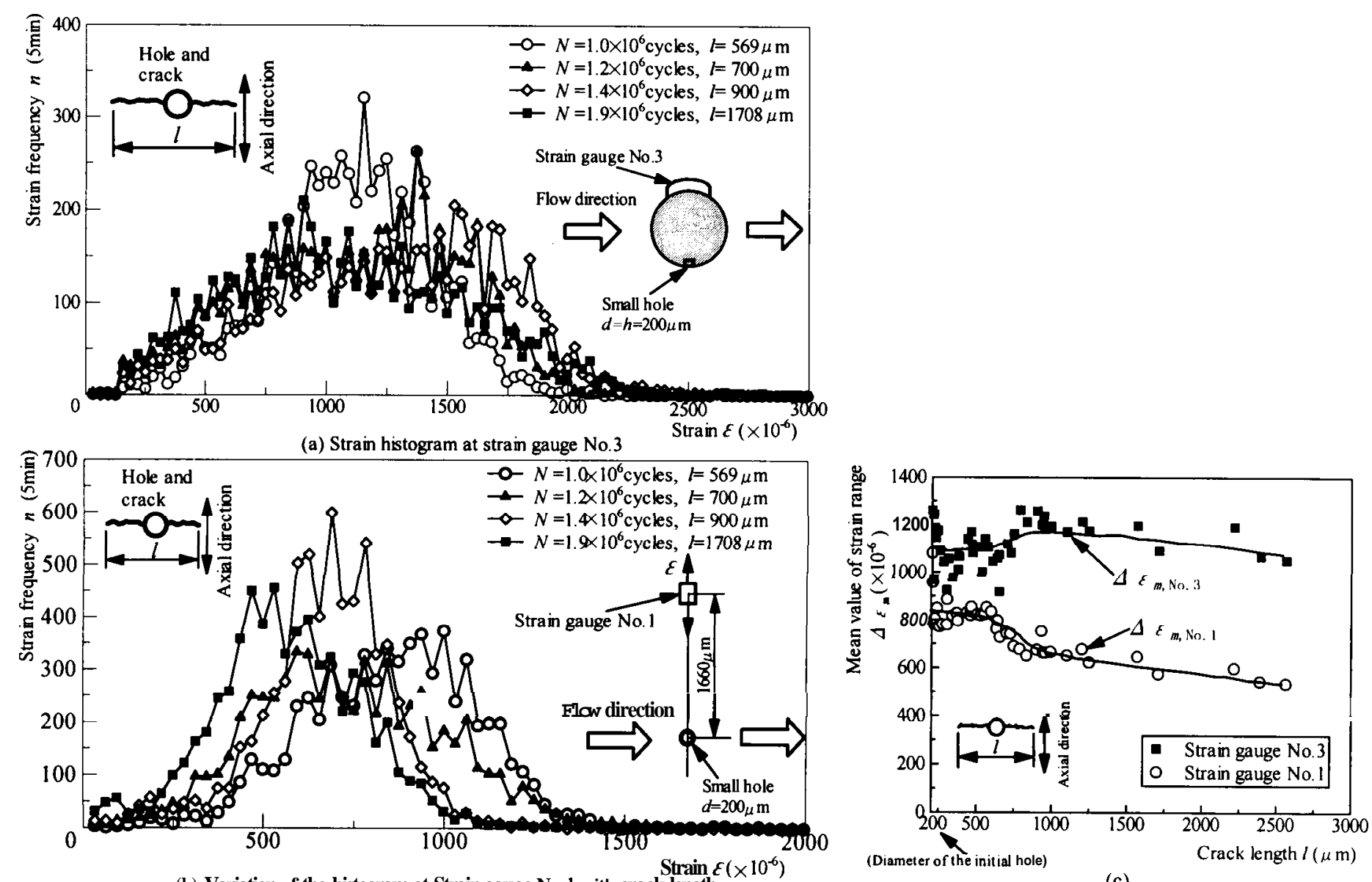

(b) Variation of the histogram at Strain gauge No.1 with crack length

(c)

Figure 7 : Histogram of strain frequency for $5 \mathrm{~min}$ measurement at various crack length. (a) Measurement at strain gauge No.1, (b) Measurement at strain gauge No.3. (c) Variation of the mean value of strain range with crack growth at strain gauges No.1 and No.3 (see Fig.3). 
varied with crack growth. On the other hand, Fig.7(b) shows the peak of strain frequency distributions at strain gauge No.1 (Fig.4(a)) shifted to the smaller value of abscissa with crack growth. It follows that the strain at strain gauge No, 1 decreased with crack growth.

For more clear detection of crack growth, a quantitative expression of variation of strain frequency distribution was attempted in relation with Fig.7(b). From strain frequency distribution of Fig.7(a) and (b), the parameter as the mean value of strain range $\Delta E_{m}$ was defined by

$$
\Delta \varepsilon_{m}=\sum_{i=1}^{256} \frac{\varepsilon_{i} n_{i}}{N_{5 \min }}
$$

where $E_{t}$ is individual strain level made by dividing $7000 \times 10^{-6}$ into 256 levels, $n$, strain frequency (reversals) and $N_{5 \min }$ total frequency (reversals) for 5 min measurement. Figure 7(c) shows that $\Delta E_{m, \text { No.3 }}$ calculated with strain frequency distribution at strain gauge No.3 is kept almost constant with crack growth. However, $\Delta E_{m, \text { No. } 1}$ at strain gauge No. 1 shows decrease of $100 \times 10^{-6}$ as crack grows from $700 \mu \mathrm{m}$ to $900 \mu \mathrm{m}$. Thus, the symptom of fatigue crack growth and fatigue failure can be captured and identified by the variation of strain near crack initiation site.

\section{Analysis of strain field near crack}

Natural frequency and displacement amplitude of the test cylinder hardly varied until final fracture. However the strain frequency distribution near the small hole shifted to smaller value approximately at crack length $1 \mathrm{~mm}$. Therefore, the change of strain field at strain gauge No. 1 with crack growth analyzed. Crack growth direction was assumed to be perpendicular to the specimen axis. Figure 8(a) shows the 2D crack model for approximation. Stresses at the strain gauge are calculated by [15]

$$
\sigma_{x}=\sigma_{y}^{\infty}\left[\frac{r}{\sqrt{r_{1} r_{2}}}\left\{\cos \left(\theta-\frac{\theta_{1}+\theta_{2}}{2}\right)-\frac{a^{2}}{r_{1} r_{2}} \sin \theta \sin \frac{3}{2}\left(\theta_{1}+\theta_{2}\right)\right\}-1\right], \quad \sigma_{y}=\frac{\sigma_{y}^{\infty} r}{\sqrt{r_{1} r_{2}}}\left\{\cos \left(\theta-\frac{\theta_{1}+\theta_{2}}{2}\right)+\frac{a^{2}}{r_{1} r_{2}} \sin \theta \sin \frac{3}{2}\left(\theta_{1}+\theta_{2}\right)\right\}
$$

where the quantities $8,8_{1}, \theta_{2}, r, r_{1}$ and $r_{2}$ are defined in Fig.8(a). With stresses $\sigma_{x}$ and $\sigma_{y}$, strain $E_{y}$ is calculated by

$$
\varepsilon_{y}=\frac{\sigma_{y}^{\infty}}{E}\left(\frac{\sigma_{y}}{\sigma_{y}^{\infty}}-v \frac{\sigma_{x}}{\sigma_{y}^{\infty}}\right)
$$

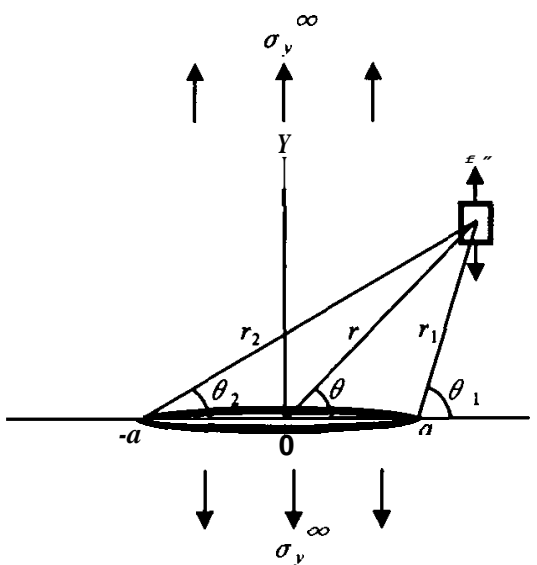

(a)

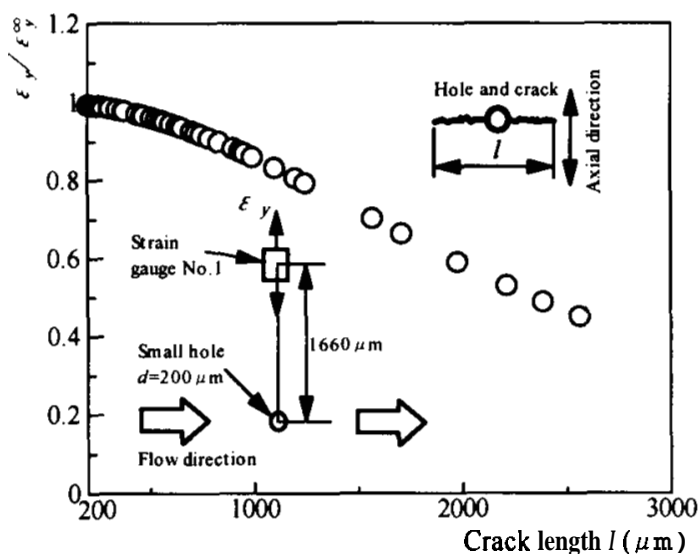

(b)

Figure 8: Analysis of strain field near 2D crack. (a) Coordinates of strain field at a crack in a two dimensional infinite plate. (b) Variation of $c / \varepsilon_{y}{ }^{\infty}$ with crack growth at strain gauge No.1. 
, where $E_{y}{ }^{\infty}=\sigma_{y}{ }^{\infty} / E, E$ Young's modulus and $v$ Poisson's ratio, 0.3. Figure 8(b) shows the change of normalized strain $\varepsilon_{y} / E_{y}{ }^{\infty}$ with crack growth. $\varepsilon_{y} / \varepsilon_{y}{ }^{\infty}$ monotonically decreases as crack grows and the decrease is evident at crack length $l=700 \mu \mathrm{m}$. The value of $\Delta E_{m, \text { No.1 }}$ in experiment also decreases as crack grows as shown in Fig.7(c). Therefore, it can be concluded that shift of strain frequency distribution to smaller value with crack growth is incorporated with relief of axial stress at the position of the strain gauge near the crack.

\section{Fatigue life evaluation based on Miner's rule}

The possibility of fatigue life evaluation by Miner's rule was examined. The total fatigue damage $D$ from the start of the test to the k-th measurement block was calculated only with first 30 min measurement data in order to examine the possibility of predicting fatigue life with the data at the start of the test. Thus, the value of $D$ is defined by

$$
D=\sum_{j=1}^{k} \sum_{i=1}^{256}\left(\frac{n_{i 1}}{N_{f i}} \times \frac{30 \mathrm{~min}}{5 \mathrm{~min}}\right)
$$

where $\mathrm{k}$ is the sequence number of measurement block, $n_{i 1}$ frequency of individual strain level in 256 divided levels of $7000 \times 10^{\prime \prime}$ during the first $30 \mathrm{~min}$ measurement, $N_{f i}$ is fatigue life for individual strain level $E, . N_{f i}$ for the same material containing an artificial small hole with diameter $200 \mu \mathrm{m}$ is formulated by Coffin-Manson type equation by [16] as

$$
\Delta E_{p}{ }^{1.77} N_{f}=0.538(0.46 \% \mathrm{C} \text { steel, } d=200 \mu \mathrm{m})
$$

, where $N_{f}=2 N_{f}$. The relationship between $N_{f}$ and total strain range $\Delta E$, can be derived by the following three equations (9), [16].

$$
\Delta \sigma / 2=1.23 \times 10^{3} \Delta \varepsilon_{p}^{0.26}, \Delta \varepsilon_{e}=\Delta \sigma / E, \quad \Delta \varepsilon_{t}=\Delta \varepsilon_{e}+\Delta \varepsilon_{p}
$$

Figure 9 shows the accumulation of fatigue damage $\mathrm{D}$ calculated by the above method with crack length which was experimentally measured. Two specimens tested in this study failed at $D$ less than unity. The cause for the value of D may be that flow-induced vibration is extremely random and includes over-stress and under-stress higher and lower than fatigue limit [17].

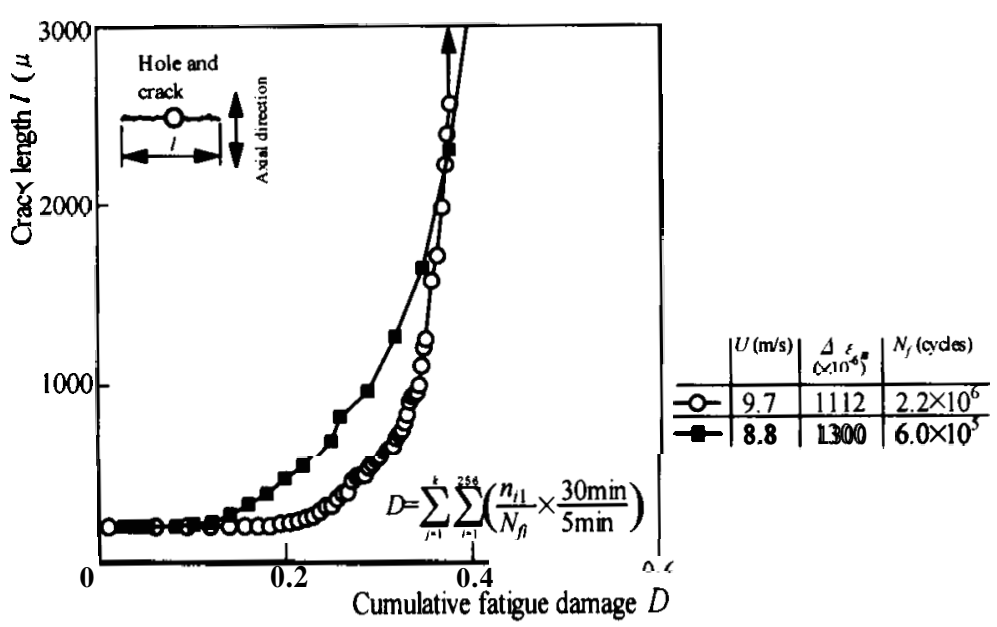

Figure 9 :Relationship between crack growth and cumulative fatigue damage. Damage curves are evaluated based on the first 30 min data 


\section{CONCLUSION}

Fatigue testing by flow-induced vibration was performed by a small wind tunnel. The conclusions obtained can be summarized as follows.

(1) Fatigue failure by flow-induced vibration transverse to flow direction occurred under Strouhal number $\mathrm{S}$ ranging from 0.10 to 0.22 .

(2) Early prediction of the symptom of fatigue failure by variation of vibration amplitude was very difficult, because the vibration amplitude of the test cylinder hardly changed until the crack grew to $1 \mathrm{~mm}$.

(3) Early prediction of the symptom of fatigue failure by variation of natural frequency was also difficult, because natural frequency did not change until the crack grew to $1 \mathrm{~mm}$.

(4) It is possible to predict the symptom of fatigue failure at crack length $1 \mathrm{~mm}$ by monitoring the variation of strain frequency distribution near crack initiation site.

(5) Fatigue damage accumulation based on Miner's rule by using the strain histogram obtained by the first $30 \mathrm{~min}$ measurement interval was $D=0.4$.

\section{REFERENCES}

1. The MONJU Website HP. http://www.jnc.go.jp/zmonju (1997). The MONJU Sodium Leak, Japan.

2. Metropolitan expressway public corporation HP. http://www.shuto-kousoku.go.jp (1999). Japan.

3. Jpn. Soc. Mech. Engnrs. ed, Ohashi, H. (1984). JSME Data Book : Failure Analysis of Machines and Structures - C a s e Histories and Techniques-, Jpn. Soc. Mech. Engnrs., p. 220.

4. Kansai Electric Power Co. LTD. Wakasa, Japan HP . http://www.kepco.co.jp (1991). Kansai Electric Power Company Inc. Milestones, Japan.

5. Iida, K. (1993). Proc. Annual Assembly of the International Institute of Welding, U.K., Doc. No. 13-1499-93.

6. Murakami, Y, Morita, T. and Mineki, K. (1997).J. Soc. Mater Sci., 46, 10, pp. 1217-1221.

7. Murakami, Y., Mineki, K., Wakamatsu, T. and Morita, T. (1997). Proc. Society of Automotive Engineers of Japan, 975, pp. 53-56.

8. Miner, M. A. (1945). J. App. Mech., 12, pp.A159-A164.

9. Robert, B. (1989). Flow-induced fibration-2nd. ed, Van Nostrand Reinhold, USA.

10. Yomiuri Newspaper Ltd, ed, Sakai, S. (1986). Document MONJU Accident, Mioshin Publisher, Japan, p. 168.

11. Anzai, H. and Endo, T. (1979).Int. J. Fatigue, 1, 1, pp. 49-57.

12. Anzai, H. (1992). The Rainflow Method in Fatigue, ed, Murakumi, Y., Butterworth Heinemann, Oxford, p. 11.

13. Chaudhari, T. and Maiti, S. (1999). Int. J. Solids \& Structures, 37, pp. 761-779.

14. Wake, R. and Evans, J. (1999). Engng. Frac. Mech., 64, pp. 177-191.

15. Okamura, H. (1976). Elementary of Linear Fracture Mechanics, Baifukan Ltd., Japan, p. 192.

16. Murakami, Y., Harada, S., Endo, T., Tani-ishi, H. and Fukushima, Y. (1983). Engng. Frac. Mech., 18, 5, pp. 909-924.

17. Nakamura, H., Horikawa, T. and Tanaka, S. (1972). Trans.Jpn. Soc. Mech. Engnrs. Ser.A, 38, pp. 683-688. 